\title{
Pool Boiling Versus Surface Wettability Characteristics
}

\author{
Fabio Villa ${ }^{1}$, Anastasios Georgoulas ${ }^{2}$, Marco Marengo ${ }^{2}$, Paolo Di Marco ${ }^{3,4}$, Joël De Coninck ${ }^{1}$ \\ ${ }^{1}$ University of Mons, Laboratory of Surface and Interfacial Physics (LPSI) \\ 19 avenue Maistriau, 7000 Mons, BE \\ fabio.villa@umons.ac.be; Joel.deconinck@umons.ac.be \\ ${ }^{2}$ University of Brighton, School of Computing, Engineering and Mathematics Department \\ Lewes Road, BN2 4GJ Brighton, UK \\ A.Georgoulas@brighton.ac.uk; M.Marengo@brighton.ac.uk \\ ${ }^{3}$ Università di Bergamo, Dipartimento di Ingegneria e Scienze Applicate \\ Viale Marconi 5, 24044 Dalmine, IT \\ ${ }^{4}$ Università di Pisa, DESTEC, Largo Lazzarino 2, 56122 Pisa, IT \\ p.dimarco@unipi.it
}

\begin{abstract}
The present paper reports results of pool boiling experiments on surfaces with different wettabilities. The main aim is to identify and quantify the difference in pool boiling characteristics (Onset of Nucleate Boiling-ONB, bubble detachment diameter and time) between Hydrophilic Surfaces (HPS) and Super-Hydrophobic Surfaces (SHS). A combined sample (SHS_D) with an inner SHS part and an outer HPS part is also tested, which clearly shows that the Super-Hydrophobic surface is a preferential area for the ONB, and allows to control the bubbling position. It is found that not only the ONB, but also the bubble growth and detachment characteristics are highly influenced by the wettability of the heated surface. The analysis of the experimental results reveals that SHSs require a lower wall-superheat for ONB to occur. Moreover, in SHSs higher bubble departure times as well as higher equivalent bubble detachment diameters are encountered. The vapour phase tends to stick stronger on a SHS than on a HPS, showing a tendency to create a vapour film. Furthermore, using a post processing method developed in MATLAB, the apparent contact angle at various successive time instances is extracted from the experimental snapshots for the cases of the HPS and the SHS_D. Finally, the capability of a previously verified, enhanced, CFD-based, VOF numerical model [1-3] to capture adequately the apparent triple line contact angle, from the nucleation up to the departure of an isolated bubble from the heated surface, is checked for one of the considered superheats for a HPS case.
\end{abstract}

Keywords: Pool boiling, wettability, ONB, bubble characteristics, VOF method, contact angle, superhydrophobicity

\section{Introduction}

In pool boiling heat is applied in a surface that is submerged in a large body of stagnant liquid. Heterogeneous boiling occurs when the temperature of the solid surface in contact with the liquid is above the saturation temperature of the liquid. As expected, the heated surface characteristics have always a corresponding influence on the boiling characteristics [4-6]. Therefore, researchers are usually focused in the optimization of the boiling surfaces [7, 8] to enhance heat transfer. For this purpose, it is highly desirable to promote an efficient boiling configuration modifying the surface characteristics in order to achieve a low temperature ONB, a high boiling heat transfer coefficient (HTC) and a high Critical Heat Flux (CHF). Various methods have been already explored in the literature so far by treating solid surfaces in order to enhance boiling characteristics. Recently Kim et al. [9] reviewed several investigations on micro/nanostructured surface design. Two main surface characteristics influence boiling (and particularly pool boiling): micro/nano-structure addition and wettability modification. The micro/nanostructure addition has a well-known influence on boiling [9-12]. A number of studies have dealt with the enhancement of boiling heat transfer from electronic components by the use of surface microstructures [13]. In the work of Kim et al. [14] the effectiveness of micro-structured surfaces (circular micropillar arrays with height, diameter and gap between 5-40 $\mu \mathrm{m}$ ) in enhancing the achieved HTCs and CHFs is investigated. It is shown that both the boiling heat transfer and critical heat flux can be enhanced (up to $+310 \%$ for CHF and $+350 \%$ for HTC) by optimising the geometrical characteristics of the micro-pillar arrays. . In Lu et al. [15] boiling experiments are conducted on a nano-porous copper surface (uniform porosity with approximately $30-200 \mathrm{~nm}$ ). It is found that on the 
nano-porous surface more active nucleation sites are present. The nano-porous structure seems to reduce the activation energy for the bubble nucleation, giving a low wall-superheat for the ONB in contrast with flat surfaces under the same heat flux conditions, as observed also in [6]. In Bourdon et al. [16] it is explained that roughness is still of importance at nanoscale (especially the mean valley depth parameter " $\mathrm{S}_{\mathrm{v}}$ " is able to quantify the sizes of the cavities present on the surface).Finally the wettability of the surface is also an influential parameter. In Fan et al. [17] the effect of surface wettability on transient pool boiling, from the super-hydrophilic to the super-hydrophobic range is studied, using a quenching method with hot stainless steel spheres. The CHF for the super-hydrophilic case was found to increase by nearly $70 \%$. The effect of the surface super-hydrophilicity is to destabilize the vapour film, helping the rewetting of the boiling surfaces even at high wall superheats. The super-hydrophobicity tends to reduce the ONB, although in default of specific micro-structured topology. In Bourdon et al. $[18,19]$ the effect of wettability on the ONB is studied systematically, on the same nano-metrically smooth surface. By grafting with different monolayers of molecules, equilibrium static contact angle is changed from $\theta_{\mathrm{eq}}=0^{\circ}$ to $\theta_{\mathrm{eq}}=110^{\circ}$ (liquid side), without changing the surface topography. A non-linear decrease of the ONB with an increase of $\theta_{\mathrm{eq}}$ is observed. Using the same experimental apparatus in [20], it is shown that in the case of a super-hydrophobic surface $\left(\theta_{\mathrm{eq}}>150^{\circ}\right)$ a quasi-Leidenfrost regime appears.

In the present paper a study of the effect of wettability on the pool boiling characteristics is carried out using the same smooth metal steel surface, untreated (HPS) and treated (SHS) by a coating to generate the necessary superhydrophobicity in order to extend the study of Bourdon et al. $[18,19]$ to the bubble characteristics, such as contact angle evolution, detachment volume and so on. In more detail, three different surface configurations are tested at various surface temperature levels of surfaces $\left(\mathrm{T}_{\text {wall }}\right)$. An untreated sample consisting of a HPS , a fully treated sample resulting in a SHS and a combine surface sample, where only a central circular part is treated while the rest remains untreated (SHS_D). From the qualitative and quantitative comparison of the results it is evident that that not only the ONB, but also the bubble growth and detachment characteristics are highly influenced by the wettability of the heated surface. The analysis of the experimental results revealed that SHSs require a lower wall-superheat for the ONB to occur. Moreover, in SHSs higher bubble departure times as well as higher equivalent bubble detachment diameters are encountered. Furthermore, the boiling process on of the HPS cases is also reproduced numerically using an enhanced VOF-based numerical model that is coupled with heat transfer and phase-change. The proposed numerical model, has already been applied, tested and validate with literature available experimental data in the past, in cases of adiabatic and diabatic bubble dynamics (in the works of Georgoulas et al. [1] and Georgoulas and Marengo [2-3]), with a high degree of convergence comparison. However, it was deemed appropriate here to further check the capability of the proposed numerical model to capture adequately also the apparent contact angle, apart from the bubble growth and detachment characteristics.

\section{Investigation Method}

\subsection{Experimental method}

The pool boiling apparatus that was used for the present investigation is described in detail in [18]. The boiling chamber is made by aluminium and several heaters are applied on it, in order to maintain a constant thermal condition for the water within the chamber. An internal heater $(80 \mathrm{~W})$ is initially used to heat up the water. Moreover, three external heating tapes are placed on the walls of the chamber and are connected to a PID controller in order to balance any potential thermal leakages. These external heating tapes work in conjunction with a cooling system (air coils), in order to raise the temperature of the chamber to the desired level and to also ensure that during the experimental test, the temperature of the chamber remains fixed (see red line in the graph of Fig. 1b) at saturation conditions (i.e. $\mathrm{T}_{\mathrm{ch}}=100^{\circ} \mathrm{C}$ and $\mathrm{P}_{\mathrm{ch}}=101.3 \mathrm{kPa}$ ). $\mathrm{T}_{\mathrm{ch}}$ and $\mathrm{P}_{\mathrm{ch}}$ are measured by a K-thermocouple and a piezo-electric pressure sensor, respectively. The sample is then heated up until the desired temperature, $\mathrm{T}_{\text {wall }}$ in Fig. $1 \mathrm{~b}$ (by an $175 \mathrm{~W}$, Acim@ JouaninH6.5X32X175 heater). Finally a heat flux meter with 3 embedded T-thermocouples is placed between the copper housing of the ceramic cartridge and the sample surface to provide a direct measure of the heat flux. All the thermocouples and the pressure gauge are connected to a PC through a data acquisition system, and the pool boiling process can be recorded, by an HCC-1000 high-speed camera (VDS Vosskuehler $\odot$, resolution of the images is $7,65 \mu \mathrm{m} / \mathrm{pixel})$. The measuring accuracy of the $\mathrm{T}$ and $\mathrm{K}$-thermocouples is $0.5 \mathrm{~K}$, accuracy of the pressure gauge is $5 \mathrm{hPa}$. 

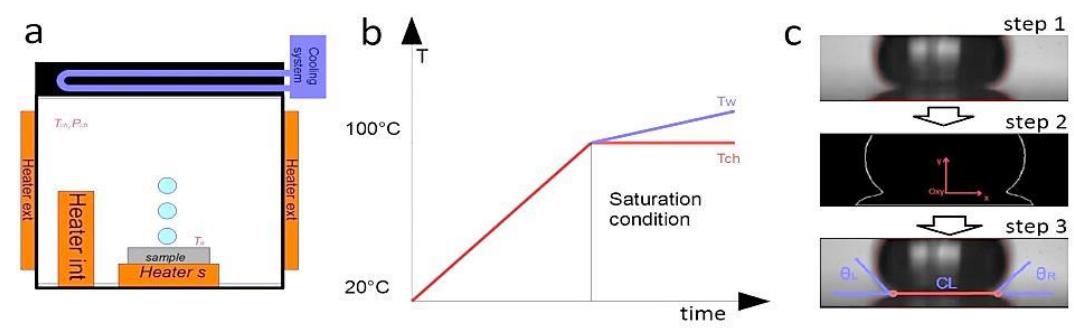

Fig. 1: a) Sketch of the experimental set-up, b) Trend of the temperature $T_{c h}$ and $T_{\text {Wall }}$ during the set-up and the test, c) Post-processing steps to measure the dynamic apparent contact angles $\left(\theta_{\mathrm{R}}\right.$ and $\left.\theta_{\mathrm{L}}\right)$ and the apparent contact line $(\mathrm{CL})$.

The wettability characteristics of the considered surfaces are summarized in Table1. As mentioned previously, three different surface configurations are tested, using these characteristics:

- A HydroPhilic Surface (HPS): AISI 316 stainless steel sample, with no coating applied.

- Super-Hydrophobic Surface (SHS): the same sample HPS is covered by a uniform and thin coating to generate super-hydrophobicity using a commercial product (Glaco Mirror Coat Zero@)

- A hybrid surface (SHS_D): only a small circle (diameter $4.6 \mathrm{~mm}$ ) in the center of the same sample HPS is covered by the SHS coating, while the rest of the sample remains hydrophilic.

Table 1: Wettability properties of the considered surfaces. Equilibrium contact angle $\left(\theta_{\text {eq }}\right)$, advancing contact angle $\left(\theta_{\text {adv }}\right)$, receding contact angle $\left(\theta_{\text {rec }}\right)$ and hysteresis $\left(\theta_{\text {adv }}-\theta_{\text {rec }}=\Delta \theta\right)$. The angles are measured using the sessile drop method. Maximum standard deviation for contact angles is $\pm 3^{\circ}$.

\begin{tabular}{|l|l|l|l|l|}
\hline & $\theta_{\mathrm{eq}}\left[{ }^{\circ}\right]$ & $\theta_{\mathrm{adv}}\left[^{\circ}\right]$ & $\theta_{\mathrm{rec}}\left[{ }^{\circ}\right]$ & $\Delta \theta\left[^{\circ}\right]$ \\
\hline HPS & 58 & 74 & 43 & 31 \\
\hline SHS & 150 & 151 & 149 & 2 \\
\hline
\end{tabular}

The roughness characteristics of the sample that is used in all the above surface configurations are summarised in Table 2. The mean roughness is quantified by the "Average Roughness" $\left(\mathrm{S}_{\mathrm{a}}\right)$ and the "Root Mean Square Roughness" $\left(\mathrm{S}_{\mathrm{q}}\right)$. However, as it is already shown in [16], the key parameter for the incipient boiling is not the mean roughness, but the peak-to-valley depth $\left(S_{z}\right)$ or even better, the "mean valley depth" $\left(S_{v}\right)$, which is able to quantify the sizes of the cavities that are present on the surface.

In order to measure the dynamic apparent contact angles of the generated bubbles $\left(\theta_{R}\right.$ and $\theta_{\mathrm{L}}$ in Fig. $\left.1 \mathrm{c}\right)$, a special post-processing routine has been developed within the general framework of Matlab@ software. The proposed image processing procedure starts from the identification of the bubble boundary (step 1 in Fig. 1c) and its digital automatic extraction to obtain the $\mathrm{X}$ and $\mathrm{Y}$ coordinates of the bubble boundary, transforming the grayscale image into a binary one (step 2 in Fig. 1c). After the identification of the solid surface (the plane $y=0$ ) a Matlab $\odot$ script automatically identifies the position of the two points where the three phases solid-liquid-vapour are in contact (in the 2D side view of the generated in each case bubble). Using a parabolic interpolation $\left(2^{\text {nd }}\right.$ order) for the shape of the bubbles close to these two points, 20 points are the selected number of point generate the proposed interpolation (a robustness tested of the image process is performed using different number of point from 5 to 30 in order to identified the optimum number of points). the apparent contact angles $\theta_{\mathrm{R}}$ and $\theta_{\mathrm{L}}$ are calculated, as the first order derivate of the parabola in the $\mathrm{y}=0$ coordinate (step 3 ). The same image post processing procedure is also applied to the numerical simulation animations of the present investigation, in order to extract the numerically predicted angles.

Table 2: Main roughness characteristics for the sample used. The reported roughness values have been measured using a confocal microscope (S neon-Sensofar@).

\begin{tabular}{|l|l|}
\hline Roughness parameter & Value $(\mu \mathrm{m})$ \\
\hline$S_{\mathrm{a}}$ & 0.046 \\
\hline $\mathrm{S}_{\mathrm{q}}$ & 0.063 \\
\hline $\mathrm{S}_{\mathrm{z}}$ & 0.989 \\
\hline $\mathrm{S}_{\mathrm{v}}$ & 0.416 \\
\hline
\end{tabular}




\subsection{Numerical method}

A brief review on available CFD-based numerical models, for the simulation of boiling heat transfer is included in the introduction section of the paper by Georgoulas and Marengo [2]. In the same work heat transfer and phase-change due to evaporation and/or condensation are coupled with a previously improved and validated Volume of Fluid (VOF) model for adiabatic bubble dynamics [1]. Moreover, the model has been validated with an existing analytical solution and with available experimental results about pool boiling. The model is then applied for the conduction of a wide range of parametric numerical experiments of pool boiling [2] as well as flow boiling within micro-channels in an accompanying paper [3]. The proposed enhanced VOF-based numerical model has been developed within the general framework of OpenFOAM, an open-source CFD Toolbox. The overall details regarding the various development and validation phases of the proposed numerical model can be found in the afore mentioned works.

In the present paper the same model is applied in order to reproduce numerically one of the HPS cases that are presented in the experimental part of the work (boiling on HPS at $\mathrm{T}_{\mathrm{w}}=117^{\circ} \mathrm{C}$ ). The aim is to further validate the proposed model by checking its ability to capture the apparent contact angle variation with time. For this purpose, a dynamic contact angle numerical treatment for the triple-line contact angle that is already implemented in OpenFOAM is also considered, apart from the constant contact angle treatment that was used before in [1-3] and the numerically predicted results from both treatments are compared with the corresponding experimental data.

Since the process of bubble growth and detachment in the proposed experiment can be considered to be axisymmetric, an axisymmetric computational domain was constructed for its numerical reproduction. A wedge type computational geometry was constructed, representing a $5^{\circ}$ section of the corresponding 3D domain in the considered physical problem. A non-uniform structured computational mesh with local refinement was used consisting of 400,000 hexahedral cells. A minimum cell size of $6 \mu \mathrm{m}$ and a maximum cell size of $50 \mu \mathrm{m}$ were selected in the bottom left and top right corners of the computational domain respectively, in order for the solution to be mesh-independent. The overall domain size in the XY plane was $10 \mathrm{~mm} \times 16 \mathrm{~mm}$. These dimensions were indicated from initial, trial simulations that were conducted in order to determine the minimum distances between the axis of symmetry and the side wall boundary (domain width) as well as between the bottom wall and the outlet (domain height), in order to avoid any influence of these boundaries in the computed bubble growth and detachment process.

At the solid walls, a no-slip velocity boundary condition was used with a fixed flux pressure boundary condition for the pressure values. At the lower wall, either a constant contact angle of $\theta_{\text {eq }}=58^{\circ}$, or a dynamic contact angle with $\theta_{\text {adv }}$ $=74^{\circ}, \theta_{\text {rec }}=43^{\circ}$ and $\theta_{\mathrm{eq}}=58^{\circ}$ is imposed for the volume fraction field. For the sidewall, a zero gradient boundary condition was used for the volume fraction values. As for the temperature field, a constant temperature of $\mathrm{T}=390.15 \mathrm{~K}$ (in accordance to the selected experimental run) was imposed in the bottom wall and a constant temperature of $\mathrm{T}=373.15 \mathrm{~K}$ was used for the sidewall (saturation temperature). At this point it should be mentioned that for the simulation a constant temperature boundary condition was used for the heated wall, while in the experiments the proposed temperature might not remain totally constant during the bubbling process. At the outlet, a fixed-valued pressure boundary condition and a zerogradient boundary condition for the volume fraction were used, while for the velocity values a special (combined) type of boundary condition was used that applies a zero gradient when the fluid mixture exits the computational domain and a fixed value condition to the tangential velocity component, in cases that fluid enters the domain. Finally, a constant value boundary condition for the temperature field, equal to the saturation value, was also prescribed at the outlet boundary. The fluid properties are kept constant.

\section{Results and Discussion}

A qualitative comparison of the bubble growth and detachment characteristics on the HPS, SHS and SHS_D surface configurations is given in Figure 2a. The overall process can be divided into 4 main stages: 1) nucleation, the generation of the bubble nucleus. At this point it should be mentioned that on the experiment we consider nucleation when the first small bubble is visible on the surface, this means that $\mathrm{t}=0 \mathrm{~s}$ for the experiment is when the bubble is already approximately $1 \mathrm{~mm}$ in diameter. However, for the numerical simulations $\mathrm{t}=0 \mathrm{~s}$ is considered at the beginning of the simulation where a small nucleus of $50 \mu \mathrm{m}$ in diameter is placed artificially on the heated plate. 2) bubble growth phase (due to evaporation), 3) bubble detachment phase, 4) waiting time phase (before the next nucleation event). 


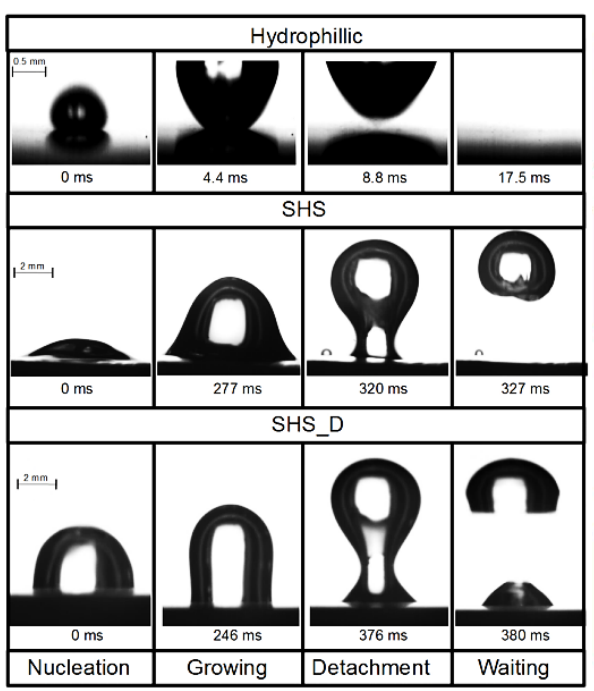

(a)

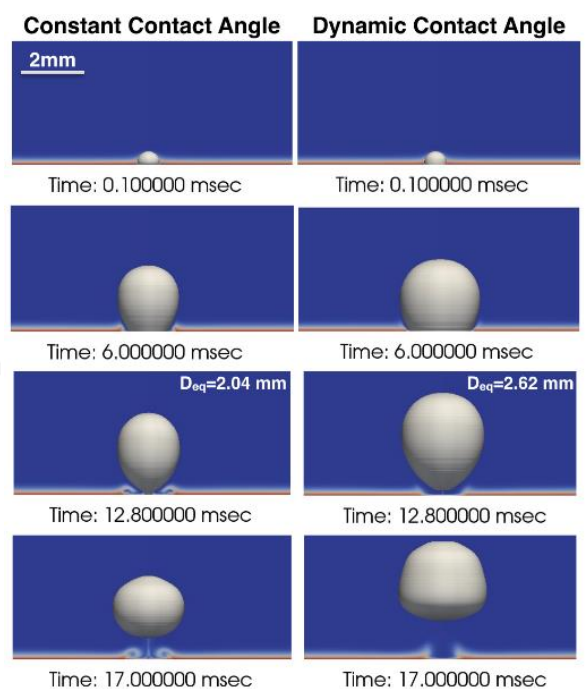

(b)

Fig. 2. (a) Sequence of the bubble growth and detachment process on the tested surfaces (HPS, SHS and SHS_D) in the experiments

(Four stages are identified: nucleation, growth, detachment, waiting time). Twall $=117^{\circ} \mathrm{C}$, saturated condition in the chamber $\mathrm{Tch}=100^{\circ} \mathrm{C} \mathrm{Pch}=1013 \mathrm{kPa}$ (b) Sequence of the bubble growth and detachment process for the HPS from the numerical reproduction of the same experimental run, with a constant contact angle (left) and a dynamic contact angle treatment (right). Twall $=117^{\circ} \mathrm{C}$, saturate condition in the chamber $\mathrm{Tch}=100^{\circ} \mathrm{C} \mathrm{Pch}=1013 \mathrm{kPa}$.

In all of these phases significant differences are observed comparing the corresponding results between the three different configurations:

- Nucleation: on the HPS boiling starts at $\mathrm{T}_{\text {wall }}=117^{\circ} \mathrm{C}$, in an arbitrary nucleation site (in the case of a completely smooth sample without any artificial cavity). On the SHS at $\mathrm{T}_{\text {wall }}=101^{\circ} \mathrm{C}$ multiple nucleation sites are initially activated that coalesce to create a vapor film on the surface, as observed also by [20] and [21]. In the case of the SHS_D, the nucleation sites appear only within the SHS circular part in the center of the sample (in a welldefined place), however no bubble nucleation occurs within the HPS part as long as $\mathrm{T}_{\text {wall }}<117^{\circ} \mathrm{C}$. Again here coalescence of initial small bubbles takes place creating a film that is bounded by the circumference of the SHS circular part.

- Growth: The bubble growing time is quite fast (approximately $10 \mathrm{~ms}$ ) on the HPS. It is possible to define a contact line and two apparent contact angles. On SHS the bubble growing time is around 10 to 20 times higher (in function of the temperature). However, it is not possible to define the contact line or the apparent contact angles in this case, since the bubbles are formed and grow directly from the initial vapor film.

- Detachment: due to the small growing time, the equivalent diameter of the detaching bubble on the HPS is also small $\left(D_{e q}=2.5 \mathrm{~mm}\right.$ for $\left.T_{w}=117^{\circ} \mathrm{C}\right)$ compared with the other two surfaces $S H S\left(D_{e q}=8.4 \mathrm{~mm}\right.$ for $\left.T_{w}=117^{\circ} \mathrm{C}\right)$ and SHS_D $\left(D_{e q}=5.4 \mathrm{~mm}\right.$ for $\left.T_{w}=117^{\circ} \mathrm{C}\right)$. The Fritz correlation [22] gives a value of $\mathrm{D}_{\text {eq }}=2.34 \mathrm{~mm}$, in good agreement with the measured value on the HPS. A significant necking stage can be noticed for the SHS and the SHS_D cases.

- Waiting: the waiting time $\left(\mathrm{t}_{\mathrm{wating}}\right)$ is the time between the detachment of a bubble and the nucleation of a new one. It is well defined only on the HPS surface, because on the SHS surface after the detachment a vapor film remains on the surface and it takes long of time to form a new growing bubble. On SHSs surfaces only the total time $\left(t_{b}\right)$ between two detachments can be evaluated.

The numerically predicted spatial and temporal evolution of the interface between the liquid and vapor phases for the case of the HPS $\left(\mathrm{T}_{\text {wall }}=117^{\circ} \mathrm{C}\right)$ is illustrated in Figure $2 \mathrm{~b}$ using the constant contact angle treatment (left) as well as the dynamic contact angle treatment (right). As it can be observed, in both cases the bubble detachment time is similar, $\mathrm{t}_{\mathrm{det}}=12.8 \mathrm{~ms}$, which is quite close to the experimentally estimated time of detachment. However, the equivalent bubble detachment diameter in the case of the dynamic contact angle treatment $\left(D_{\text {eq }}=2.62 \mathrm{~mm}\right)$ is much more closer to the 
experimentally calculated value $\left(D_{\text {eq }}=2.50 \mathrm{~mm}\right)$ as well as to the theoretical correlation of Fritz [22] $\left(D_{\text {eq }}=2.34 \mathrm{~mm}\right)$ than in the case of the constant contact angle treatment $\left(\mathrm{D}_{\mathrm{eq}}=2.04 \mathrm{~mm}\right)$.

Fig. 3 depicts the experimentally derived bubble detachment frequency $\left(\mathrm{f}_{\mathrm{b}}\right)$ on the HPS (Fig. 3a), SHS and SHS_D (Fig. 3b) at different wall temperatures $T_{w}$. The frequency $f_{b}$ is defined as:

$$
\overline{f_{b}}=\frac{1}{N} \sum_{i=1}^{N} \frac{1}{t_{b}^{i}}
$$

Where $\mathrm{t}_{\mathrm{b}}{ }^{\mathrm{i}}$ is the time between two consecutive detachment events and $\mathrm{N}$ is the total number of detachment events in one video. So $t_{b}$ is the sum of the growing time (for the bubble $i$ ) and the waiting time (time between the detach of the bubble $i$ and the nucleation of the next bubble $i+1)$. Using this definition a comparison between the HPS and the SHS cases is feasible in terms of the bubble frequency, since on the SHS cases it is not possible to identify the start of the nucleation, but only the detachment of the bubble. There is clearly an increasing trend in the bubble detachment frequency with the corresponding increase in the heated wall temperature $T_{\text {wall }}$. This is primarily due to the corresponding reduction of the waiting time [23] and also due to the reduction of the detachment time. It is also characteristic that the $\mathrm{f}_{\mathrm{b}}$ value on the HPS cases is about 10 times bigger than in the cases of the SHS and the SHS_D.
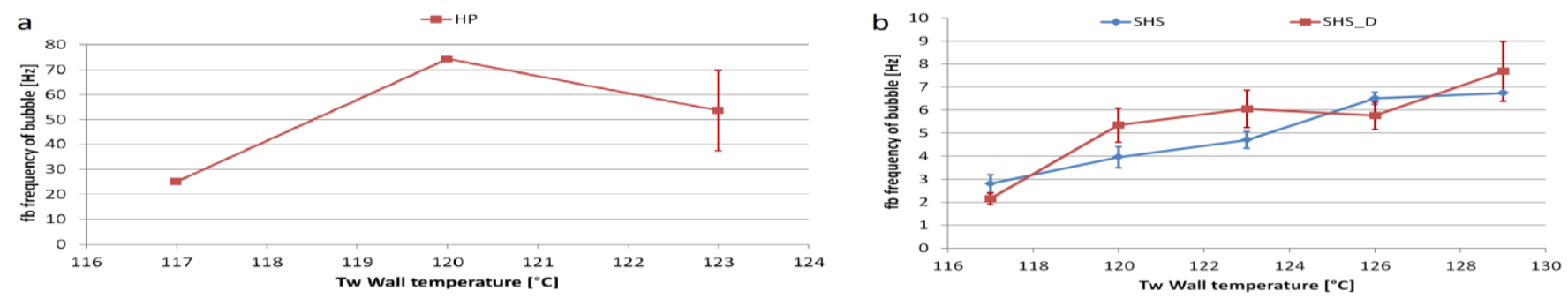

Fig. 3: a) Frequency $f_{b}$ for the HPS in function of $T_{\text {wall }} b$ ) Frequency $f_{b}$ for the SHS and the SHS_D in function of $T_{\text {wall }}$.

In Figure $4 \mathrm{a}$, the time evolutions of the apparent dynamic contact angles $\theta_{\mathrm{R}}$ and $\theta_{\mathrm{L}}$ for the HPS case are plotted. As it can be observed, the apparent contact angle remains in the range $\theta_{\text {adv }}<\theta_{\mathrm{R}}<\theta_{\text {rec }}$ during the bubble growth process (at higher tested temperature $\mathrm{T}_{\mathrm{w}}=123^{\circ} \mathrm{C}$ seems lower then $\theta_{\text {rec }}$ for $\mathrm{t}<3 \mathrm{~ms}$ ). Before the detachment, $\theta_{\mathrm{R}}$ appears to be closer to $\theta_{\text {adv }}$ (due to symmetry, the same consideration can be done for $\theta_{\mathrm{L}}$ ). The $\theta_{\text {adv }}$ is the equilibrium contact angle during the expansion of liquid on the surface (as evaluated using the drop sessile method), that correspond on the CL reduction phase (liquid re-wetting the surface) before detachment. Apparent contact angles can be evaluated also on SHS_D. $\theta_{\mathrm{R}}$ slightly oscillates around $90^{\circ}$ (the bubble grows primarily in the vertical direction as shown in Fig. 2) until the formation of a neck that induces a final increase before detachment. In the case of the HPS it is quite evident that the variation of the heated wall temperature has a noticeable effect in the apparent contact angles, especially in the initial expanding stage of the CL. However, not a clear increasing or decreasing trend can be observed with respect to the corresponding increase in the temperature of the heated wall.
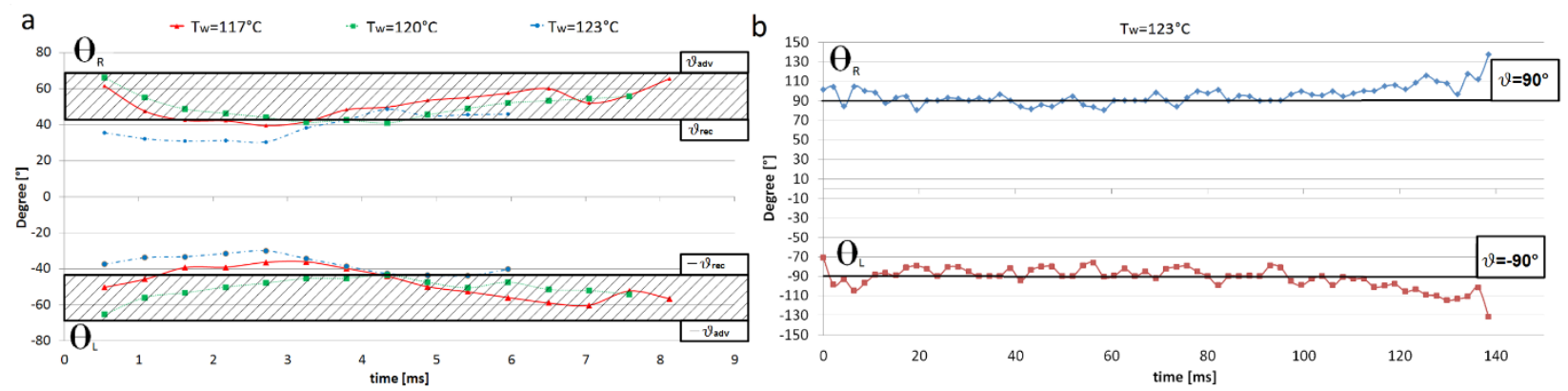

Fig. 4: a) Apparent dynamic contact angles $\theta_{R}$ and $\theta_{L}$ for HPS surface at different $T_{w}$. b) Apparent dynamic contact angles $\theta_{R}$ and $\theta_{L}$ for SHS_D surface at $\mathrm{T}_{\mathrm{w}}=123^{\circ} \mathrm{C}$. 
A comparison of the dynamic, apparent contact angles between the experiment (red line) as well as the numerical simulations with the constant contact angle treatment (green line) and the dynamic contact angle treatments (blue line), is made in Figure 5, for the case of $\mathrm{T}_{\text {wall }}=117^{\circ} \mathrm{C}$. The apparent contact angle is evaluated in the simulation using the same image processor, applied to the images on Fig. $2 \mathrm{~b}$.

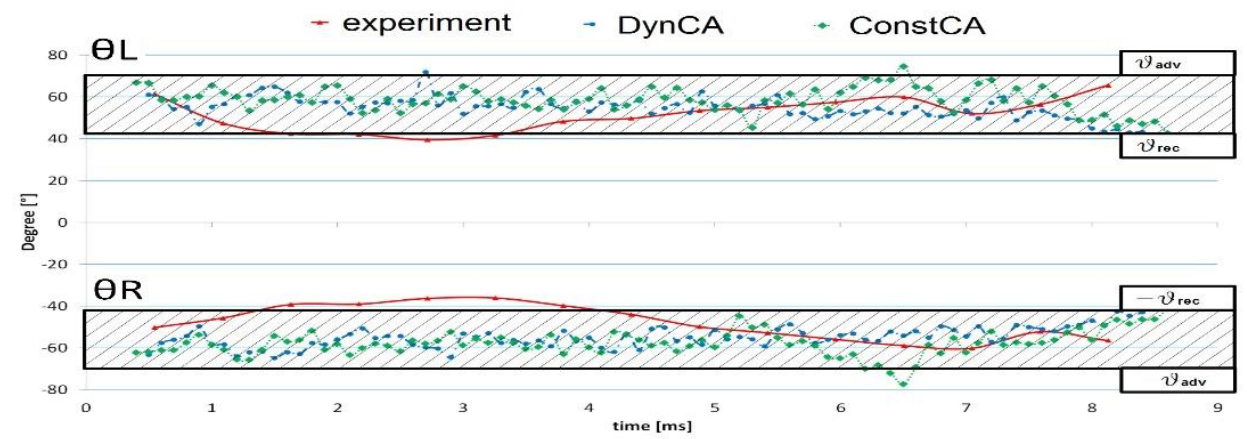

Fig. 5: a) Apparent dynamic contact angles $\theta_{\mathrm{R}}$ and $\theta_{\mathrm{L}}$ for HPS surface. $\mathrm{T}_{\text {wall }}=117^{\circ} \mathrm{C}$, saturate condition in the chamber $\mathrm{T}_{\mathrm{ch}}=100^{\circ} \mathrm{C}$ $\mathrm{P}_{\mathrm{ch}}=1013 \mathrm{kPa}$. Comparison of the experimental measurements with the corresponding numerical predictions for both the constant as well as the dynamic contact angle treatments.

As it can be observed the dynamic contact angle treatment is again more close to the experimental curve, especially at the last stage of the bubble growth, before its detachment from the heated plate. Therefore, it is evident that the dynamic contact angle treatment gives better results regarding both the bubble detachment characteristics as well as the apparent contact angle revolution with time, in comparison to the corresponding experimental data.

\section{Conclusions}

The effect of wettability on the pool boiling characteristics is investigated in the present paper, carrying out experiments on three different surfaces with varying given wettability characteristics (HPS, SHS and SHS_D) and varying different heated wall superheats. Furthermore, one of the HPS cases is also reproduced numerically using an enhanced VOF-based numerical model that is coupled with heat transfer and phase-change [1-3]. This is done in order to further check the ability of the proposed numerical model to capture dynamic bubble growth characteristics, such as the time evolution of the apparent contact angles, apart from the bubble detachment time and the equivalent bubble detachment diameter. For this purpose two different numerical treatments for the triple-line contact angle are used (a static/constant contact angle and a dynamic contact angle treatment) and the results are compared with the corresponding experimental data. From the overall analysis and discussion of results the following worth mentioning conclusions can be withdrawn:

$>$ Comparing the experimental runs to each other, it is evident that not only the ONB, but also the bubble growth and detachment characteristics are highly influenced by the wettability of the heated surface. The analysis of the experimental results revealed that SHSs require a lower wall-superheat for the ONB to occur. Moreover, in SHSs higher bubble departure times as well as higher equivalent bubble detachment diameters are encountered.

> From the overall comparison of the numerical simulations with the corresponding experimental data it is evident that the dynamic contact angle treatment for the triple contact line (solid, vapor, liquid) gives more accurate predictions with respect to the equilibrium contact angle input for both the bubble detachment characteristics as well as for the dynamic evolution of the apparent contact angle.

\section{References}

[1] A. Georgoulas, P. Koukouvinis, M. Gavaises, and M. Marengo, "Numerical investigation of quasi-static bubble growth and detachment from submerged orifices in isothermal liquid pools: The effect of varying fluid properties and gravity levels," International Journal of Multiphase Flow, vol. 74, pp. 59-78, 2015.

[2] A. Georgoulas and M. Marengo, "Numerical simulation of pool boiling: the effects of initial thermal boundary layer, contact angle and wall superheat," in Proceedings of the UK Heat Transfer Conference, Edinburgh, 2015. 
[3] A. Georgoulas and M. Marengo, "Numerical simulation of flow boiling in micro-channels: bubble growth, detachment and coalescence," in Proceedings of the UK Heat Transfer Conference, Edinburgh, 2015.

[4] Y. Qi, J. F. Klausner, and R. Mei, "Role of surface structure in heterogeneous nucleation," International Journal of Heat and Mass Transfer, vol. 47, pp. 3097-3107, 2004.

[5] J. P. McHale and S. V. Garimella, "Nucleate boiling from smooth and rough surfaces - Part 2: Analysis of surface roughness effects on nucleate boiling," Experimental Thermal and Fluid Science, vol. 44, pp. 439-455, 2013.

[6] C. Young Lee, M. M. Hossain Bhuiya, and K. J. Kim, "Pool boiling heat transfer with nano-porous surface," International Journal of Heat and Mass Transfer, vol. 53, pp. 4274-4279, 2010.

[7] M. Zupančič, M. Steinbücher, P. Gregorčič, and I. Golobič, "Enhanced pool-boiling heat transfer on laser-made hydrophobic/superhydrophilic polydimethylsiloxane-silica patterned surfaces," Applied Thermal Engineering, vol. 91, pp. 288-297, 2015.

[8] A. Jaikumar and S. G. Kandlikar, "Enhanced pool boiling heat transfer mechanisms for selectively sintered open microchannels," International Journal of Heat and Mass Transfer, vol. 88, pp. 652-661, 2015.

[9] D. E. Kim, D. I. Yu, D. W. Jerng, M. H. Kim, and H. S. Ahn, "Review of boiling heat transfer enhancement on micro/nanostructured surfaces," Experimental Thermal and Fluid Science, vol. 66, pp. 173-196, 2015.

[10] H. S. Ahn, C. Lee, J. Kim, and M. H. Kim, "The effect of capillary wicking action of micro/nano structures on pool boiling critical heat flux," International Journal of Heat and Mass Transfer, vol. 55, pp. 89-92, 2012.

[11] L. Dong, X. Quan, and P. Cheng, "An experimental investigation of enhanced pool boiling heat transfer from surfaces with micro/nano-structures," International Journal of Heat and Mass Transfer, vol. 71, pp. 189-196, 2014.

[12] H. Jo, J. M. Kim, H. Yeom, G. C. Lee, H. S. Park, M. Kiyofumi, et al., "Boiling performance and material robustness of modified surfaces with multi scale structures for fuel cladding development," Nuclear Engineering and Design, vol. 291, pp. 204-211, 2015.

[13] H. Honda and J. J. Wei, "Enhanced boiling heat transfer from electronic components by use of surface microstructures," Experimental Thermal and Fluid Science, vol. 28, pp. 159-169, 2004.

[14] S. H. Kim, G. C. Lee, J. Y. Kang, K. Moriyama, M. H. Kim, and H. S. Park, "Boiling heat transfer and critical heat flux evaluation of the pool boiling on micro structured surface," International Journal of Heat and Mass Transfer, vol. 91, pp. 1140-1147, 2015.

[15] L. Lu, T. Fu, Y. Tang, T. Tang, B. Tang, and Z. Wan, "A novel in-situ nanostructure forming route and its application in pool-boiling enhancement," Experimental Thermal and Fluid Science, vol. 72, pp. 140-148, 2016.

[16] B. Bourdon, R. Rioboo, M. Marengo, E. Gosselin, and J. De Coninck, "Influence of the wettability on the boiling onset," Langmuir, vol. 28, pp. 1618-1624, 2012.

[17] L.-W. Fan, J.-Q. Li, D.-Y. Li, L. Zhang, and Z.-T. Yu, "Regulated transient pool boiling of water during quenching on nanostructured surfaces with modified wettability from superhydrophilic to superhydrophobic," International Journal of Heat and Mass Transfer, vol. 76, pp. 81-89, 2014.

[18] B. Bourdon, P. Di Marco, R. Rioboo, M. Marengo, and J. De Coninck, "Enhancing the onset of pool boiling by wettability modification on nanometrically smooth surfaces," International Communications in Heat and Mass Transfer, vol. 45, pp. 11-15, 2013.

[19] B. Bourdon, E. Bertrand, P. Di Marco, M. Marengo, R. Rioboo, and J. De Coninck, "Wettability influence on the onset temperature of pool boiling: Experimental evidence onto ultra-smooth surfaces," Advances in Colloid and Interface Science, vol. 221, pp. 34-40, 2015.

[20] I. Malavasi, B. Bourdon, P. Di Marco, J. de Coninck, and M. Marengo, "Appearance of a low superheat "quasiLeidenfrost" regime for boiling on superhydrophobic surfaces," International Communications in Heat and Mass Transfer, vol. 63, pp. 1-7, 2015.

[21] I. U. Vakarelski, N. A. Patankar, J. O. Marston, D. Y. C. Chan, and S. T. Thoroddsen, "Stabilization of Leidenfrost vapour layer by textured superhydrophobic surfaces," Nature, vol. 489, pp. 274-277, 2012.

[22] S. Hamzekhani, M. Maniavi Falahieh, and A. Akbari, "Bubble departure diameter in nucleate pool boiling at saturation: Pure liquids and binary mixtures," International Journal of Refrigeration, vol. 46, pp. 50-58, 2014.

[23] R. Situ, M. Ishii, T. Hibiki, J. Y. Tu, G. H. Yeoh, and M. Mori, "Bubble departure frequency in forced convectiv subcooled boiling flow," International Journal of Heat and Mass Transfer, vol. 51, pp. 6268-6282, 2008. 